\title{
Evaluation of Performance of Fuzzy C Means and Mean Shift based Segmentation for Multi-Spectral Images
}

\author{
Sandeep Kaur \\ Research Scholar \\ CSE Department \\ GIMET
}

\author{
Shikha Chawla \\ Assistant Professor \\ CSE Department \\ GIMET
}

\begin{abstract}
Image Segmentation has become very useful vision application because it can be used in many image processing applications. An image segmentation results in an images where each object is differentiated from other one. Many segmentation techniques have been proposed so far to get accurate segmentation results. This paper has focused on Mean Shift and Fuzzy C means clustering algorithm to segment multispectral images in more accurate manner.
\end{abstract}

\section{Keywords}

Image Segmentation, Clustering, Mean Shift, FCM.

\section{INTRODUCTION}

Generally Digital images are collection of pixels and these pixels remain prearranged now a systematic rectangular display. The dimensions of this pixel array are used for the determination of the magnitude of an image. The image width and height is determined from the number of columns and the number of rows in the array. Image coordinates $\mathrm{x}$ and $\mathrm{y}$ are defined for any specific pixel within image matrix. Bits are castoff to express the intensity values of the image and bit has only two possible values 0 or 1 . The aim of image segmentation is to divide an image into expressive sections per respect to a specific use. It is based on some properties like grey level, intensity, texture, colour etc. It is beneficial on behalf of image study and explanation; the sections should intensely narrate to illustrated objects of attentiveness. Reliability of segmentation is very important for the success of image analysis and a very stimulating difficulty in image segmentation is accurate partitioning. There are various methods which are used for image segmentation are: mean shift based segmentation, minimum spanning tree based segmentation, clustering based segmentation, region based segmentation, edge detection based segmentation etc.

\section{CLUSTERING}

A collection of objects which are alike between them and are unalike to the objects belonging to other clusters is called clustering. It can also be defined as the course of forming entities addicted to sets whose followers are alike in some means. Image clustering addresses some issues like: 1) In what way to signify the image. 2) In what way to establish the facts. 3) In what way to categorize an image toward a definite group. Different types of clustering methods are available like: k-means clustering, fuzzy-c means, Mean Shift based clustering etc. From all these methods now we describe fuzzyc means and Mean Shift based methods as following.

\subsection{Mean Shift based Segmentation}

Mean Shift is a progressive method for segmentation based on clustering. In this method we do not have need of prior knowledge of how many number of clusters are needed because it is a non-parametric iterative method. Kernel Density Estimation which is a non-parametric way to estimate the width of the Parzen window [14]. Mean shift defines a window around each data point and calculates the mean of data point. Then it shifts the center of window to the mean and repeats the algorithm till it converges. This process is appropriate in over segmentation, multiple segmentation, tracking, clustering applications [13]. The basic mean shift algorithm is discussed in following points.

Step 1: For each data point

1) Designates a structure or window nearby point.

2) Then estimates the mean of data point.

3) Then moves the focus of window towards the mean and replications the procedure till it merging.

\subsection{Fuzzy-C Means method}

The FCM clustering algorithm was first introduced by Dunn and later was extended by Bezdek [15].The procedure is an iterative clustering method FCM is a data clustering method now which a dataset is assembled addicted to $\mathrm{n}$ groups by each data opinion now the dataset be appropriate to every single group toward a certain point. Example for this like, a certain data point that deceits near toward the center of a group will require a high degree of be appropriate to that group and a different data point that deceits distant away from the center of a cluster will have a low degree of belonging or membership to that cluster.

The next is the illustration of stages involved in this clustering method [15]:

Step 1: Firstly guess for the centers of the cluster. The initial guess for centers of cluster is mostly to be improper.

Step 2: FCM assigns a membership to every data point for every cluster.

Step 3: FCM iteratively changes the cluster centers toward the accurate position inside a specific data set. This iteration is created on decreasing an impartial purpose that signifies the space from some certain data point toward a cluster center biased via that data point's membership rating.

\section{LITERATURE SURVEY}

Choong, et al,2013][1] has discussed clustering algorithm in Normalised Cuts based image segmentation in this evaluation of the clustering algorithm with the normalised cuts image segmentation on images has been carried out and the effect of different image complexity towards normalised cuts segmentation process is presented. Dhara, Bibhas Chandra, et al. [2011][2] has discussed color feature is used to degree the familiarity among two areas and therefore the tag 
of the unmarked area is decided. Zhou, et al. [2008] [3] has discussed a mean shift based fuzzy c-means algorithm for image segmentation. Mean shift can rapidly and dependably bargain cluster midpoints; the whole approach is skilled of optimally segmenting clusters inside an image. Zhang, et al. [2008] [8] has discussed a 2D histogram prediction remains castoff to precise the Otsu threshold. The 1D histograms remain developed through $2 \mathrm{D}$ histogram estimate cutting-edge $\mathrm{x}$ and $\mathrm{y}$ axes and a debauched algorithm aimed at penetrating the great of the projected histogram is planned created on the wavelet transmute is here descried. Tentative consequences display that the projected way does well than the old Otsu method aimed at our renal biopsy examples. Chenyi Xia, et al. [2006] [4] has discussed on identifies the boundary points from the data sets and also uses one of the database techniques called Gorder $\mathrm{kNN}$ join and also use a special property of the reverse $k$ nearest neighbour $(\mathrm{RkNN})$. This study has proved that BORDER is able to find boundary points efficiently and effectively and increase the accuracy of clustering. Pal, et al. [2005] [5] has discussed a model called possibilistic-fuzzy c-means. PFCM resolves the noise compassion fault of FCM overwhelms the parallel clusters difficult of PCM and rejects the row amount restraints of FPCM. Comaniciu, et al. [2003] [6] has discussed a growth of a consistent procedure which proceeds addicted to explanation the constancy of local bandwidth approximations through measures. The rationality of the hypothetical consequences is confirmed in numerous space separating tests including the variable-bandwidth mean shift. A.K. Jain, et al.[1999] [12] has discussed an imprint of strategy clustering methods commencing a statistical plan acknowledgment perspective, by a part of given that respected material and circumstances to significant thoughts obtainable to the wide community of clustering specialists. They present index of clustering approaches, and classify cross-cutting subjects and present growths.

\section{EXPERIMENTAL SETUP}

In order to evaluate the Mean Shift and Fuzzy $\mathrm{C}$ means based segmentation the MATLAB has been used. To evaluate these segmentation techniques various tools available in the MATLAB.

\section{RESULTS}

To evaluate the performance of Mean Shift and Fuzzy C means based segmentation.

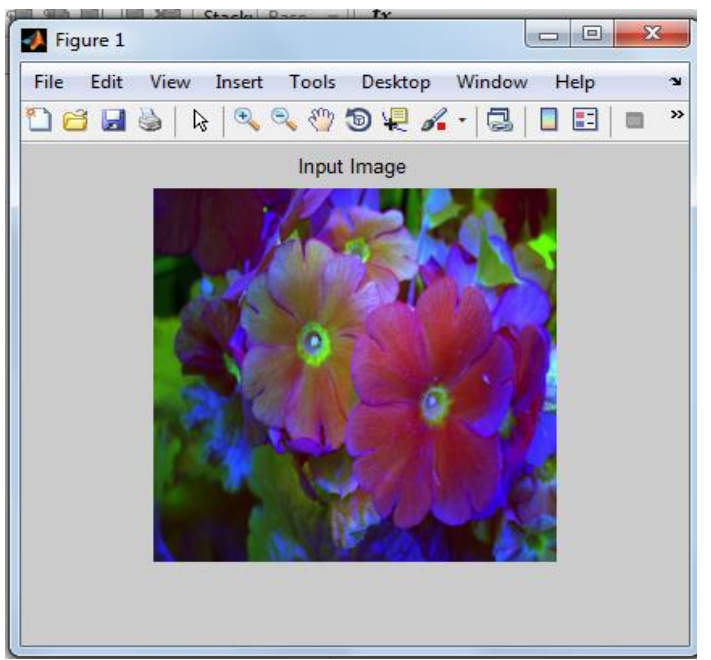

Figure 1: Input Image
Figure 1 shows the input image which will be used for the evaluation of Mean Shift and Fuzzy C means based Segmentation.

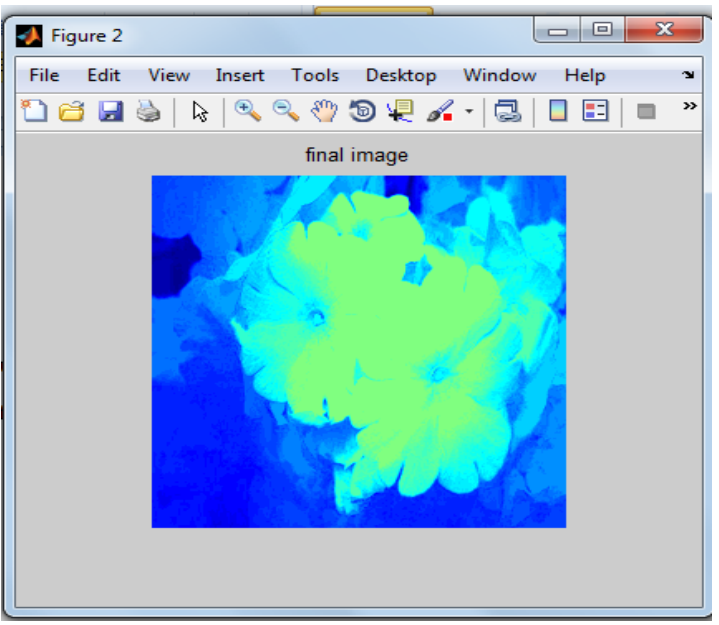

Figure 2: Image from Mean Shift based Segmentation

Mean Shift and Fuzzy C means based segmentation algorithms are applied separately on the input images. Figure 2 shows the image obtained from the Mean shift based segmentation algorithm.

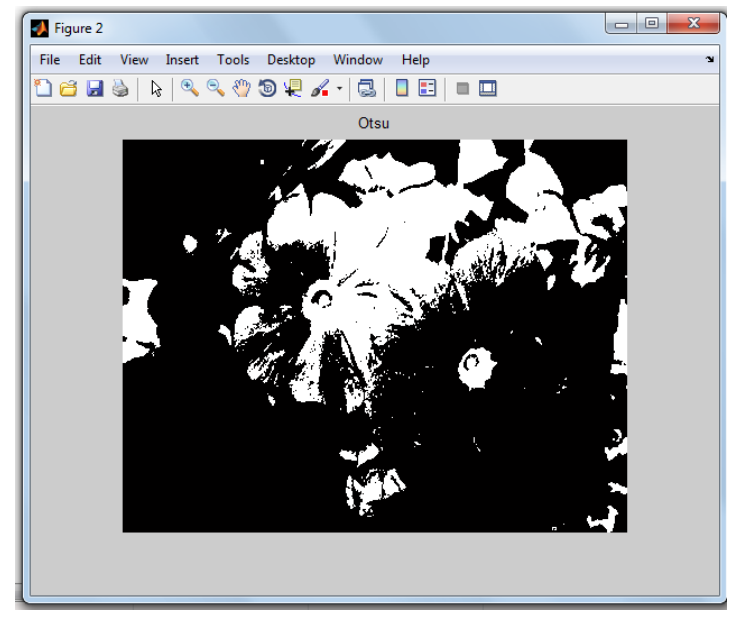

Figure 3: OTSU obtained from fuzzy $\mathrm{C}$ means algorithm

Figure 3 shows the OTSU image is firstly obtained when Fuzzy $\mathrm{C}$ means based segmentation algorithm is applied on the input image.

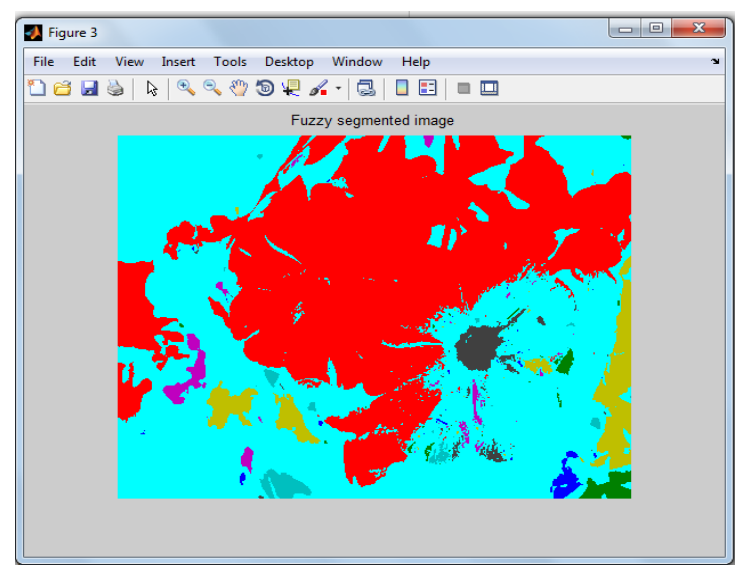

Figure 4: Fuzzy segmented image is obtained 
Figure 4 shows the Fuzzy segmented image when fuzzy C means algorithm applied on the input image.

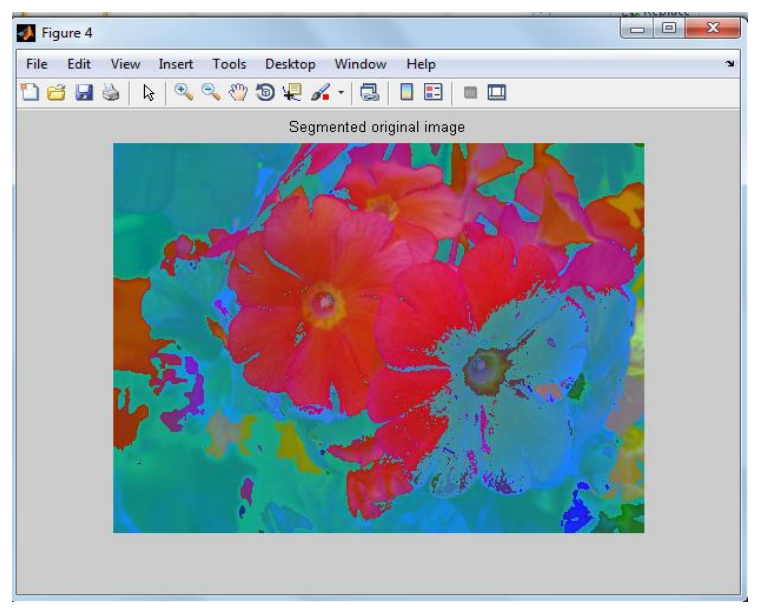

Figure 5: Original Segmented Image

Figure 5 shows the final original segmented image obtained using Fuzzy $\mathrm{C}$ means algorithm.

\section{PERFORMANCE EVALUATION}

The table 1 displays the outcomes of evaluation of Mean Shift and Fuzzy $\mathrm{C}$ means based segmentation methods on the centre of Mean Squared Error using at least nine images rather in result section we show results by taking only one image. Mainly MSE specifies the regular alteration of the pixels all over the image. If MSE is higher than it demonstrate a larger variance among the original and processed image.

Table 1: Mean Squared Error Analysis

\begin{tabular}{|c|c|c|}
\hline Image & $\begin{array}{c}\text { Mean } \\
\text { Shift }\end{array}$ & FCM \\
\hline IMG 1 & 0.1363 & 0.1369 \\
\hline IMG 2 & 0.1909 & 0.1733 \\
\hline IMG 3 & 0.2927 & 0.1657 \\
\hline IMG 4 & 0.2799 & 0.1932 \\
\hline IMG 5 & 0.2653 & 0.1836 \\
\hline IMG 6 & 0.1921 & 0.1934 \\
\hline IMG 7 & 0.3324 & 0.1996 \\
\hline IMG 8 & 0.2613 & 0.2378 \\
\hline IMG 9 & 0.1881 & 0.0798 \\
\hline
\end{tabular}

The above table shows the evaluation results of the Mean Shift and FCM algorithm based on Mean Squared Error Analysis.

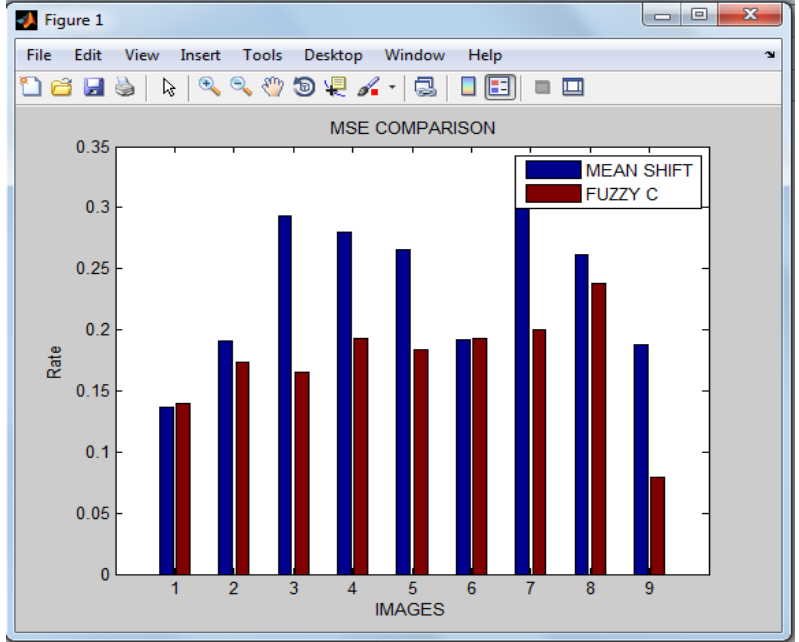

Figure 6: Mean Squared Error Analysis

The graph attained on behalf of MSE of these images is exposed in Figure 6 below and the result shows that mean shift based algorithm attains highest values as compared to Fuzzy c means based segmentation algorithm.

The table 2 displays the outcomes of evaluation of Mean Shift and Fuzzy $\mathrm{C}$ means based segmentation methods on the centre of Peak Signal to Noise Resolution. The PSNR calculates the peak signal-to-noise ratio, in decibels, among binary images. This proportion is repeatedly castoff as a superiority capacity among the original and a resultant image. The higher the PSNR, the better is the quality of the output image.

Table 2: Peak Signal to Noise resolution Analysis

\begin{tabular}{|c|c|c|}
\hline Image & Mean Shift & FCM \\
\hline IMG1 & 28.3129 & 54.3704 \\
\hline IMG2 & 27.4744 & 53.7979 \\
\hline IMG3 & 28.4178 & 55.9712 \\
\hline IMG4 & 26.9689 & 55.3053 \\
\hline IMG5 & 27.2874 & 55.5272 \\
\hline IMG6 & 26.4689 & 55.3004 \\
\hline IMG7 & 34.1203 & 55.1641 \\
\hline IMG8 & 27.3168 & 54.4028 \\
\hline IMG9 & 26.2672 & 59.1475 \\
\hline
\end{tabular}

The above table shows the evaluation results of the Mean Shift and FCM algorithm based on Peak Signal to Noise Resolution and the values of the table shows that FCM contains highest values for PSNR as compared to Mean Shift algorithm. 


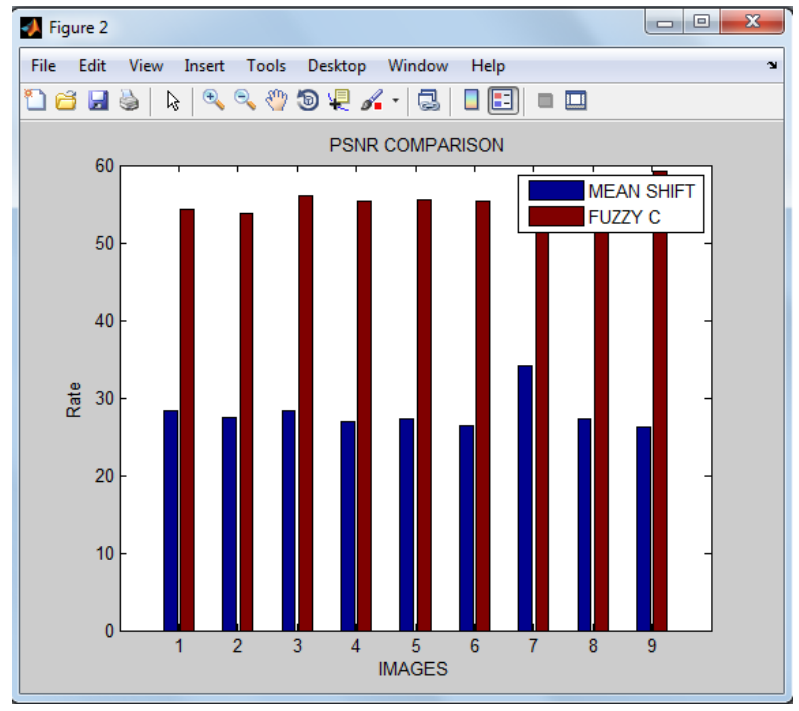

Figure 7: PSNR Comparison

The graph attained on behalf of PSNR of these images is exposed in Figure 7 below and this figure shows that FCM gives higher value as compared to Mean Shift Based Segmentation.

\section{CONCLUSION AND FUTURE SCOPE}

Segmentation divides the image into numerous sections according to their features like gray level, color, texture, intensity etc. and these sections cover the whole image or represent the whole image. FCM springs well consequences for overlapped datasets because in this technique each data point not restricted to particular cluster rather than data point can belong to more than one cluster according to the degree of membership. Mean Shift is a method that preserves the discontinuity of the images. This paper has implemented Fuzzy C means and Mean Shift based segmentation in MATLAB with the help of image processing tool box. Comparative analysis has shown that the Fuzzy $\mathrm{C}$ means provides more accurate results than Mean Shift algorithm because it contains highest values for PSNR parameter and almost contains lowest values for MSE parameter. However this research work has not considered any improvement in existing segmentation techniques. So in near future we will propose a new algorithm which will integrate Fuzzy C means, Modified Mean Shift with Minimum Spanning Tree based algorithm.

\section{REFERENCES}

[1] Choong, Mei Yeen, Wei Leong Khong, Renee Ka Yin Chin, Farrah Wong, and Kenneth Tze Kin Teo. 2013 "Clustering Algorithm in Normalised Cuts Based Image Segmentation." In Modelling Symposium, 2013 7th Asia, pp. 166-171. IEEE, 2013.

[2] Dhara, Bibhas Chandra, and Bhabatosh Chanda. 2011 "A Fast Interactive Image Segmentation to Locate Multiple Similar-Colored Objects." IEEE Third National Conference on Computer Vision, Pattern Recognition, Image Processing and Graphics, pp. 25-28., 2011.

[3] Zhou, Huiyu, Gerald Schaefer, and Chunmei Shi. 2008 "A mean shift based fuzzy c-means algorithm for image segmentation." In Engineering in Medicine and Biology Society, 2008. EMBS 2008. 30th Annual International Conference of the IEEE, pp. 3091-3094. IEEE, 2008.

[4] Zia, Chenyi, Wynne Hsu, Mong Li Lee, and Beng Chin Ooi. 2006 "BORDER: Efficient Computation of Boundary Points." Knowledge and Data Engineering, IEEE Transactions on 18, no. 3 (2006): 289-303.

[5] Pal, Nikhil R., Kuhu Pal, James M. Keller, and James C. Bezdek. 2005 "A possibilistic fuzzy c-means clustering algorithm." Fuzzy Systems, IEEE Transactions on 13, no. 4 (2005): 517-530.

[6] Comaniciu, Dorin. 2003 "An algorithm for data-driven bandwidth selection." Pattern Analysis and Machine Intelligence, IEEE Transactions on 25, no. 2 (2003): 281288 .

[7] Georgescu, Bogdan, Ilan Shimshoni, and Peter Meer. "Mean shift based clustering in high dimensions: A texture classification example." In Computer Vision, 2003. Proceedings. Ninth IEEE International Conference on, pp. 456-463. IEEE, 2003.

[8] Zhang, Jun, and Jinglu Hu. 2008 "Image segmentation based on 2D Otsu method with histogram analysis." In Computer Science and Software Engineering, 2008 International Conference on, vol. 6, pp. 105-108. IEEE, 2008.

[9] Kanzawa, Yuchi, Yasunori Endo, and Sadaaki Miyamoto. 2011 "On hard and fuzzy c-means clustering with conditionally positive definite kernel." In Fuzzy Systems (FUZZ), 2011 IEEE International Conference on, pp. 816-820. IEEE, 2011.

[10] Wang, Weina, Yunjie Zhang, Yi Li, and Xiaona Zhang. 2006 "The global fuzzy c-means clustering algorithm." In Intelligent Control and Automation, 2006. WCICA 2006. The Sixth World Congress on, vol. 1, pp. 36043607. IEEE, 2006.

[11] Liu, Dongju, and Jian Yu. 2009 "Otsu method and Kmeans." In HIS'09. Ninth International Conference on, vol. 1, pp. 344-349. IEEE, 2009.

[12] Wain, Anil K., M. Narasimha Murty, and Patrick J. Flynn. 1999 "Data clustering: a review." In ACM computing surveys (CSUR) 31.3 (1999): 264-323

[13] Randhawa, Amanjot Kaur, and Rajiv Mahajan. 2014 "An Improved Approach towards Image Segmentation Using Mean Shift and FELICM." International Journal of Advanced Research in Computer Science and Software Engineering 4, no. 7 (2014): 197-202.

[14] Banerjee, Biplab 2014 "Unsupervised Multi-Spectral Satellite Image Segmentation Combining Modified Mean-Shift and a New Minimum Spanning Tree Based Clustering technique.",In IEEE Journal of 7.3 (2014): 888-894

[15] Detection, Segmentation Using Sub Pixel. "Fuzzy Based Hyperspectral Image Segmentation using Sub Pixel Detection." International Journal of Information 4, no. 3 (2014). 